\title{
Advances in Conformal Antennas
}

\author{
K. Sumalatha, Ch. R. Phani Kumar
}

\begin{abstract}
In wireless communication technology, due to fast advancement use of small size antenna has rapidly increased. Not only the antenna size, but also its cost, performance, eases of installation everything have been taken care while designing the antenna. To meet these entire requirement conformal antennas is proposed. Conformal antennas have wide applications in commercial, defense and several civil, systems. In aircrafts and ships they are the need hour and most essential. For such conformal antennas the Patch antennas are often considered as the better candidate. In this paper, we discuss the conformal antennas with various substrates used for designing the antenna. Through this paper, we develop a modeling of conformal antennas with recent advances through surveying of early manifestations.
\end{abstract}

Keywords: Conformal antennas, wireless communication technology, Patch antennas, commercial, defense

\section{INTRODUCTION}

The conformal antenna is a radiating framework according to the IEC (International Electro-technical Commission), by its electromagnetic features whose shape isn't resolved $[1,2]$. By the framework surface with the headway in novel methods, methodologies and innovation to the system configuration are must, where it must be admission. In free space, an ordinary radiating system alludes to a system which goes about as interface between the receiver and transmitter. So as to enhance the overall system performance it is conceivable to change the characteristics. On a few aerodynamic systems this antenna can be introduced. Alongside conformal nature they have to group's broadband, miniaturization and multiband characteristics. Conformal antenna has a place with the phased array antenna class. They comprise an array of little flat antennas like patches and dipoles surface covering.

In numerous applications conformal antennas are utilized where arrangement of remarkable antenna is required. This may incorporate the antenna integration on circular arrays for more extensive inclusion [3, 4], on an aircraft or vehicle [5-7], for load-bearing purposes unique composites for implanting the antenna into a structure [8], for systems being integrated in wearable wireless network as textile antennas [9-11]. The antenna systems are required to work with progressively adaptive beam-forming capabilities, efficiency for greater radiation, structural robustness and cost effective $\mathrm{RF}$ electronics for a large number of these applications. Besides, in complex radiation environment which incorporate vibrating or changing surfaces and multiple radiators these prerequisites are to be met.

Revised Manuscript Received on 14 August, 2019.

Mrs. K. Sumalatha, Assoc.Professor, Dept. of ECE, Malla Reddy Engineering College for Women(Autonomous), Secunderabad-TS kadire.suma@gmail.com

Dr. Ch.R.Phani Kumar, Asst. Professor, GITAM University, Vizag, Andhra Pradesh, India, rphanikumar.chintalapudi@gmail.com
Numerous new research regions on embroidered RF circuits [12], E-textile conductors [13], and metalized fibers [14] have been produced due to the complex idea of these applications. From these endeavors the outcomes have been exceptionally valuable and huge numbers of the issues related with physically implementing conformal antennas have tended to. Because of unwanted surface deformations, the radiation properties of these conformal antennas can be seriously degraded [15]. Because of their capacity for high gain, beam shaping, and beam steering the antenna array has pulled in increasingly more consideration as of late. To synthesize the ideal radiation pattern in antenna array pattern synthesis the fundamental concern is to discover proper excitation phase and amplitude.

To take care of complex antenna pattern synthesis issues different strategies including optimization algorithms and traditional mathematical systems have been introduced [16, 17]. For the most part for linear homogeneous array the traditional mathematical system is utilized. The conformal arrays elements by and large direct their radiation beams toward various bearings in curved surfaces and to the desired pattern not all elements contribute similarly. In pattern synthesis this characteristic outcomes in challenges in pattern synthesis.

The radiation pattern can be enhanced as the shape of conformal antenna changes [18], past research work has demonstrated that with proper passive and active mechanical dampening, mechanical steering, phase compensation and voltage amplitude, and actuators. The focus of this research is to survey the current procedures, methodologies, tools and frameworks of conformal antenna for surface changes, polarization and pattern synthesis. Rest of the paper is sorted as follows: section 1 includes the introduction part. Segment 2 discusses the various modeling of conformal antenna. Finally, section 3 concludes the paper.

\section{MODELING OF CONFORMAL ANTENNA}

Considering the recent advancements in the wireless technology, there are numerous approaches to design conformal antenna. This section glances at different existing techniques. The surveys were selected to incorporate the current procedures and methods applied to different information for design of conformal antenna. Examined techniques and methods are within the past recent years that fall in categories of changing surfaces, polarization and pattern synthesis techniques. Figure 2 shows the modeling of conformal antenna with various methods.

Braaten et al. [19] have exhibited a phased-array test stage for concentrate the self-adapting capabilities of conformal antennas. Alongside four individual printed micro-strip patch antennas connected to a conformal surface, the authors designed a four-port receiver (2.45-GHZ) with voltage controlled attenuators and phase 
shifters. With a flexible SMA Cable every antenna was associated with the comparing receiver port. The distorted radiation pattern of the array can be recouped with proper phase compensation as conformal array changes surface. To build up another self-adapting flexible microstrip antenna array with an embedded flexible sensor system this pattern recovery data was utilized. To gauge the surface deformation of the conformal array and to control the phase shifters the sensor circuit was utilized.

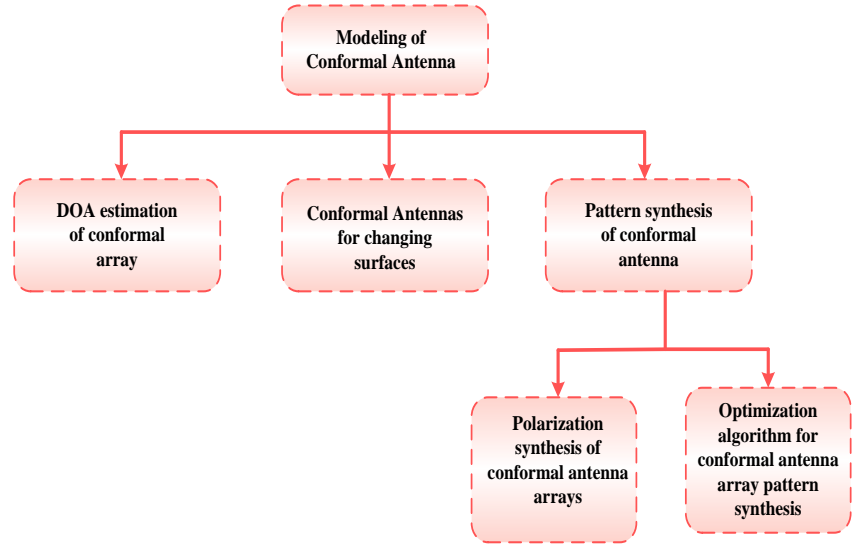

Figure 2: Modeling of conformal antenna

\subsection{Modeling of Conformal Antennas for Changing Surfaces}

Cheng et al. [20] exhibited a millimeter-wave shaped-beam substrate integrated conformal array antenna. In the wake of examining the conformal shape impact on the characteristics of a radiating slot and a Substrate Integrated Waveguide (SIW), on a cylindrical surface the array mounted with a radius of $20 \mathrm{~mm}$ is synthesized at the center frequency (35 $\mathrm{GHz}$ ). In a solitary dielectric substrate all components, including a phase compensated network, $8 \times 8$ slot array and 1-to- 8 divider were created together. At the beam direction the cross polarization was lower than $-41.7 \mathrm{db}$. In millimeter-wave frequency band, the presented SIW conspire had the capacity to illuminate the trouble of reconciliation between a feed network and conformal array elements. From the feed network, while maintaining a strategic distance from element-to-element parasitic cross-coupling and spillage in radiation.

Self-adapting conformal antennas for changing spherical surfaces was researched by the creators in [21]. In particular, the theory on the relationship between the element spacing of the conformal array, required phase compensation and spherical surface radius was produced. For theoretical approval, with individual microstrip antennas utilized as the radiators at $2.47 \mathrm{GHz}$ at first a $4 \times 4$ phased array antenna was collected. To a financially accessible voltage controlled phase shifter with indistinguishable SMA cables every antenna was associated and afterward to a port on a sixteen-way power divider each phase shifter was connected. On the spherical surface this phased-array antenna takes into account convenient placement of individual patches. To quantify the radius of curvature of the spherical surface the sensing circuit was utilized. This data use to autonomously apply the suitable phase compensation to recoup the radiation pattern of the array for various spherical surfaces at 2.47 GHz dependent on the past theoretical improvements.
To changing conformal surfaces Muhammad Saeed Khan et al. [22] was created another $1 \times 4$ frequency reconfigurable self-adapting conformal antenna array. The displayed conformal array comprises of four reconfigurable elements of microstrip patch antenna. To quantify the voltage controlled phase shifters and the curvature of conformal surface a reconfigurable sensor circuit was utilized. By the reconfigurable sensing circuit these phase shifters were controlled. The phase compensation was actualized to autonomously recuperate the pattern in the two bands of the reconfigurable; the array was appended upon shape change. A conformal wide-band SIW H-plane horn antenna mounted on a substantial conducting cylinder was displayed by Yun Zhao et al. [23]. When it was mounted on a conducting cylinder of shifting radius the impact of a curved low-profile SIW H-plane horn was inspected. The two air slots along the sides of the flare part of the antenna were sliced so as to compensate the crumbling of the VSWR of the H-plane horn antenna. The matching between the horn antenna and the feed line was enhanced with the slots use and at the same time the working frequency bandwidth was broadened. In their work from $6.1 \mathrm{GHZ}$ to $19 \mathrm{GHZ}$ the VSWR measured of the fabricated antenna was below 2.5. over the same frequency range the antenna likewise shows stable radiation.

\subsection{DOA Estimation of Conformal Array}

For DOA estimation of conformal array, several algorithms have already presented [24-28].

In a Massive Multiple-Input-Multiple-Output (MIMO) framework under obscure mutual coupling, a novel 2D DOA estimation approach dependent on the tensor method was presented by Xiaoyu Lan et al. [24] for a conformal array. The declaration of received signal was defined with the Khatri Rao (KR) product by setting sensors uniformly on the cylindrical surface. In light of the conformal array an obscure mutual coupling auto-suppression technique was researched. To use the multidimensional data of the received information dependent on the conformal array signal model a third-order tensor was constructed. By HOSVD (Higher-Order Singular Value Decomposition) moreover the signal subspace was given. At long last, by conventional subspace-based algorithms the DOAs were estimated.

Based on conformal array a 2-D DOA estimation algorithm for coherently distributed (CD) sources was presented by Liangtian Wan et al. [25]. In view of GSVS (Generalized Steering Vectors) three rational invariance relationships were built. For estimating three rational invariance matrices then the PM (Propagator Method) was utilized. At last, from the Eigen values of three rational invariance matrices the 2-D DOA of CD sources were gotten. The presented algorithm have low computational complexity without Eigen decomposition of sampling covariance matrix and searching of spectrum peaking and estimation.

A novel high accuracy 2D-DOA estimation algorithm for the conformal array was introduced by Liangtian Wan et al. [26]. By means of PARAFAC (Parallel Factor Analysis) theory a 2D-DOA estimation of the cylindrical conformal exhibit was practiced. Due to the polarization diversity of the fluctuating curvature the customary DOA estimation algorithm can't be utilized on conformal array. In the covariance domain the algorithm shapes a PARAFAC model of the covariance 
matrices to stay away from the issue of parameter pairing. To other conformal array structures and non-uniform noise scenario the presented algorithm was likewise summed up. To confirm the execution of the presented algorithm the simulation results with the cylindrical conformal array were introduced and the CRB (Cramer-Rao Bound) was derived. A conformal cylindrical microstrip antenna array was planned and explored by the authors in [27]. To investigate the superior of 2-D DOA estimation, A NC-MUSIC (non-circular music) algorithm with a conformal cylindrical array was incorporated. For every element in the antenna array the electric fields were dissected and considered the impacts of it on NC-MUSIC algorithm. Their reenactment results demonstrates that the presented technique have expanded the most extreme estimation number of NC-MUSIC algorithm and have higher resolution than the standard music algorithm.

In ITS (Intelligent Transportation Systems) with conformal antenna, a mutual coupling coefficient and a novel doa estimation algorithm was presented by Yan Zou et al. [28]. Alongside instrumental element technique the impact of mutual coupling was wiped out by developing the special MCM (mutual coupling matrix). In view of PARAFAC theory, the DOA of incident signals was estimated. In cumulate domain utilizing covariance matrices the PARAFAC demonstrate was developed. In light of the matrix transformation between the steering vector and MCM the mutual coupling coefficients were evaluated.

\subsection{Pattern Synthesis of Conformal Antenna}

In this section the pattern synthesis of conformal antenna based on optimization algorithms and polarization with different approaches is discussed.

\subsubsection{Optimization Algorithm for Conformal Antenna Array Pattern Synthesis}

For pattern synthesis of conformal antenna arrays Zhipeng Liang et al. [29] have presented a hybrid GA-PSO algorithm (Genetic Algorithm and Particle Swarm Optimization). At the point when connected to antenna array pattern syntheses utilizing the two strategies advantages, the introduced method have high convergence accuracy and fast convergence speed. A few average test functions and optimization instances of a linear array pattern synthesis were shown to demonstrate the execution of the hybrid optimization algorithm. To exhibit the presented algorithm at last a $4 \times 2$ cylindrical conformal microstrip antenna array as a down to earth synthesis precedent was examined. For conformal antenna array pattern synthesis, estimated and reenacted results have demonstrated the introduced method was reliable and compelling.

Within the sight of necessities on both the DRR (Dynamic Range Ratio) of excitations and the polarization, Comisso and Roberto Vescovo [30] have displayed an iterative algorithm for the 3D synthesis of the electric far-field pattern of a conformal antenna array. In a given angular area the displayed calculation permits a versatile control of the polarization and of the DRR. To give the array excitations it requires a low time of CPU. Also to empower the optimization of the polarization state by phase-only control a changed adaptation of the algorithm was created. For the joint polarization and pattern synthesis of conformal arrays with lessened DRR numerical outcomes were displayed to confirm the helpfulness of the exhibited methodology.

Karimzadeh Baee et al. [31] tended to conformal array synthesis as an obliged issue of multi objective optimization. With a constraint on fundamental beam direction, synchronous decrease of XPL (cross-polarization level) and SLL (side lobe level) was pointed. To optimize the pattern a hybrid of WARP (weighted alternating reverse projection) and 2LB-MOPSO (Two Local Best Multi-Objective Particle Swarm Optimization) was presented. In their research at first the WARP method finds a feasible and moderate solution. At that point 2LB-MOPSO technique starts, incorporating the WARP solution with an underlying population and the capacities for handling constraint penalty. In the underlying population of 2LB-MOPSO including WARP result leads to evading local extreme traps, less sensitivity and higher convergence rate to the functions of penalty. After adequate iterations the presented method gives better SLL and XPL when Compared to WARP technique which quickly stagnates.

In [32], for conformal antenna arrays a quick strategy that empowers the synthesis of cross polar patterns, 3D co-polar, simultaneously decreasing the DRR of the array excitations was presented. By presenting two auxiliary phase patterns in particular, one for the co-polar pattern and the other for the cross polar pattern the power synthesis issue was decreased to a field synthesis one. Concerning the array excitations and two auxiliary phase patterns the issue was then iteratively settled. For the particular case the altered version of the technique was introduced where the decrease of DRR isn't required, yet the computational time was additionally lessened.

To advance the element layout for conformal arrays Yang et al. [33] have introduced a technique. With its location the element pattern in a conformal array shifts inferable from the impact of platform. To reenact the element patterns at all conceivable locations it was normally extremely time consuming. Utilizing the model-based parameter estimation (MBPE) strategy by interpolating the element patterns at a few sampled locations, the patterns of an element situated at a subjective position on the platform were gotten in the exhibited technique. There are two stages in interpolation methodology. At the sampled locations it interpolates the Fourier coefficients of the given element patterns and then to get the element pattern the IFFT/IDFT (Inverse Fast Fourier Transform/Inverse Discrete Fourier Transform) algorithm was connected. By the PSO strategy the element layout was optimized.

Ouyang et al. [34] have synthesized and investigated a conformal conical surface linear phased array with enhanced NSGA-II algorithms and VSIE (volume-surface integral equation). With the VSIE algorithm the port characteristic and element patterns of antenna with mutual coupling have been reenacted. By their characteristic impedances named active element patterns, all elements patterns were ended and for correlation that were measured in a microwave chamber. Between the measured and the reproduced outcomes their outcomes demonstrate a decent match. With different weights of all these active patterns of elements the pattern of the conformal antenna array was a superposition. In any case, the 
objects configuration containing the levels of side lobe, direction of beam and 3-db beam width was considered. In their work, for a multi-object optimization the INSGA-II algorithm was utilized.

In pattern synthesis of conformal arrays Yan-Ying Bai et al. [35] have presented a hybrid IWO (invasive weed optimization) and PSO (particle swarm optimization) algorithm. To show the presented algorithm a cylindrical conformal microstrip array was considered. Utilizing the hybrid IWO/PSO algorithm to accomplish the required objectives the amplitude and phase of each excited voltage were optimized and the active element pattern of every element in the conformal array was extricated. Exploratory result shows while barring their separate lacks the presented hybrid algorithm keeps up the particular advantages of the IWO and PSO calculations when contrasted and standard PSO and IWO algorithms. In addition, in accomplishing global optimality the presented hybrid algorithm was very reliable.

A convex Optimization based full polarimetric sum and difference patterns synthesis technique for a conformal array was presented by Zhijiang Huang et al. [36]. To demonstrate the conformal array reaction the strategy receives a co-polarization, co-polarization and a manifold separation procedure from exhibit calibration measurements. At that point, plan the convex Optimization issues as patterns synthesis of polarization sum and difference. To a reproduced conformal spherical conformal array made out of 176 antenna elements the technique was connected and the low cross-polarization level difference and sum patterns and low side lobe were presented.

\section{2.3.2 POLARIZATION SyNTHESIS OF CONFORMAL ANTENNA ARRAYS}

To synthesize matched dual polarization patterns for accurate polarimetric radar measurement Wanqiu $\mathrm{Hu}$ et al. [37] have connected a convex optimization theory TO conformal antenna arrays. In a convex shape the creators have figured the issue of optimization and the parameters assurance strategy was displayed. To affirm the potentiality of the presented strategy, the two array configurations; cylindrical and spherical arrays with crossed dipoles and dual-polarized patches elements were introduced.

The structure of sparse conformal phased array with elements situated on a truncated cone shape was tended to by the creators in [38]. Of an air traffic control system the antenna, supposed to be a fully-digital receiver, so as to cover a desired angular region it need to at the same time produce multiple receiving beams along the elevation plane. So as to characterize the set of complex excitations for every individual beam and the positions of the array elements, by methods for the Multi-Task Bayesian Compressive Sensing (MTBCS) the synthesis issue was completed. To approve the adequacy of the displayed technique a preliminary numerical result was reported. The summary of the reviewed methods and techniques is shown in table 1.

\section{CONCLUSION}

This paper analyses the various approaches and schemes that is utilized in conformal antenna arrays design. It can be concluded that, these models are based only on the convex optimization theory based synthesis. Particular conformal patch antenna can be designed for specific applications. By changing surface, polarization and pattern synthesis the presented models can be improved and yield more accurate forecasts. The pattern synthesis of conformal antenna became much fast and efficient while using optimization techniques. So, an enhancement to the design of conformal antenna along with hybrid optimization algorithm can be proposed as a future work.

\section{REFERENCES}

1. Polegre, G. Caille, L. Boyer and A. Roederer, "Semi-active conformal array for ESA's GAIA mission", IEEE Antennas and Propagation Society Symposium, 2004., 2004.

2. $\mathrm{Y}$. $\mathrm{Wu}$ and $\mathrm{Y}$. Cheng, "Conical Conformal Shaped-Beam Substrate-Integrated Waveguide Slot Array Antenna With Conical-to-Cylindrical Transition", IEEE Transactions on Antennas and Propagation, vol. 65, no. 8, pp. 4048-4056, 2017.

3. H. Schippers, "Radiation analysis of conformal phased array antennas on distorted structures", 2018.

4. H. Schippers, J. Verpoorte, P. Jorna, A. Hulzinga, A. Meijerink, C. Roeloffzen, R. Heideman, A. Leinse and M. Wintels, "Conformal phased array with beam forming for airborne satellite communication", 2008 International ITG Workshop on Smart Antennas, 2008.

5. L. Vaskelainen, "Phase synthesis of conformal array antennas", IEEE Transactions on Antennas and Propagation, vol. 48, no. 6, pp. 987-991, 2000 .

6. H. Schippers, J. Verpoorte, P. Jorna, A. Hulzinga, A. Meijerink, C. Roeloffzen, R. Heideman, A. Leinse and M. Wintels, "Conformal phased array with beam forming for airborne satellite communication", 2008 International ITG Workshop on Smart Antennas, 2008.

7. L. Vaskelainen, "Phase synthesis of conformal array antennas", IEEE Transactions on Antennas and Propagation, vol. 48, no. 6, pp. 987-991, 2000.

8. P. Li, W. Xu, L. Song and Y. Qiu, "A Novel Inversion Method of Manufacturing Flaws in the Packaging of Conformal Load-Bearing Antenna Structure", 2018.

9. M. Klemm and G. Troester, "Textile UWB Antennas for Wireless Body Area Networks", IEEE Transactions on Antennas and Propagation, vol. 54, no. 11, pp. 3192-3197, 2006.

10. T. Kennedy, P. Fink, A. Chu, N. Champagne, G. Lin and M. Khayat, "Body-Worn E-Textile Antennas: The Good, the Low-Mass, and the Conformal", IEEE Transactions on Antennas and Propagation, vol. 57 , no. 4, pp. 910-918, 2009

11. P. Salonen, Y. Rahmat-Samii, M. Schaffrath and M. Kivikoski, "Effect of textile materials on wearable antenna performance: a case study of GPS antennas", IEEE Antennas and Propagation Society Symposium, 2004. 2004.

12. Zheyu Wang, Lanlin Zhang, Y. Bayram and J. Volakis, "Multilayer printing of embroidered RF circuits on polymer composites", 2011 IEEE International Symposium on Antennas and Propagation (APSURSI), 2011.

13. Y. Bayram, Y. Zhou, B. Shim, S. Xu, J. Zhu, N. Kotov and J. Volakis, "E-Textile Conductors and Polymer Composites for Conformal Lightweight Antennas", IEEE Transactions on Antennas and Propagation, vol. 58, no. 8, pp. 2732-2736, 2010.

14. S. Morris, Y. Bayram, L. Zhang, Z. Wang, M. Shtein and J. Volakis, "High-Strength, Metalized Fibers for Conformal Load Bearing Antenna Applications", IEEE Transactions on Antennas and Propagation, vol. 59, no. 9, pp. 3458-3462, 2011.

15. K. Wincza and S. Gruszczynski, "Influence of Curvature Radius on Radiation Patterns in Multibeam Conformal Antennas", 2006 European Microwave Conference, 2006

16. [16]G. Oliveri, P. Rocca and A. Massa, "Differential evolution as applied to electromagnetics: Advances, comparisons, and applications", 2012 6th European Conference on Antennas and Propagation (EUCAP), 2012.

17. $\mathrm{Xu}$, "Robust adaptive neural control of flexible hypersonic flight vehicle with dead-zone input nonlinearity", Nonlinear Dynamics, vol. 80, no. 3, pp. 1509-1520, 2015.

18. J. Helander, K. Zhao, Z. Ying and D. Sjoberg, "Performance Analysis of Millimeter-Wave Phased Array Antennas in Cellular Handsets", IEEE Antennas and Wireless Propagation Letters, vol. 15, pp. 504-507, 2016. 
19. Braaten, S. Roy, S. Nariyal, M. Al Aziz, N. Chamberlain, I. Irfanullah, M. Reich and D. Anagnostou, "A Self-Adapting Flexible (SELFLEX) Antenna Array for Changing Conformal Surface Applications", IEEE Transactions on Antennas and Propagation, vol. 61, no. 2, pp. 655-665, 2013.

20. Y. Cheng, H. Xu, D. Ma, J. Wu, L. Wang and Y. Fan, "Millimeter-Wave Shaped-Beam Substrate Integrated Conformal Array Antenna", IEEE Transactions on Antennas and Propagation, vol. 61, no. 9, pp. 4558-4566, 2013.

21. B. Braaten, S. Roy, I. Irfanullah, S. Nariyal and D. Anagnostou, "Phase-Compensated Conformal Antennas for Changing Spherical Surfaces", IEEE Transactions on Antennas and Propagation, vol. 62, no. 4, pp. 1880-1887, 2014.

22. M. Khan, B. Ijaz, D. Anagnostou, B. Braaten, A. Capobianco and S. Asif, "Frequency reconfigurable self-adapting conformal array for changing surfaces", IET Microwaves, Antennas \& Propagation, vol. 10, no. 8, pp. 897-901, 2016

23. Y. Zhao, Z. Shen and W. Wu, "Conformal SIW H-Plane Horn Antenna on a Conducting Cylinder", IEEE Antennas and Wireless Propagation Letters, vol. 14, pp. 1271-1274, 2015.

24. X. Lan, L. Wang, Y. Wang, C. Choi and D. Choi, "Tensor 2-D DOA Estimation for a Cylindrical Conformal Antenna Array in a Massive MIMO System Under Unknown Mutual Coupling", IEEE Access, vol. 6, pp. 7864-7871, 2018.

25. L. Wan, G. Han, J. Jiang, C. Zhu and L. Shu, "A DOA Estimation Approach for Transmission Performance Guarantee in D2D Communication", Mobile Networks and Applications, vol. 22, no. 6, pp. 998-1009, 2017.

26. L. Wan, W. Si, L. Liu, Z. Tian and N. Feng, "High Accuracy 2D-DOA Estimation for Conformal Array Using PARAFAC", International Journal of Antennas and Propagation, vol. 2014, pp. 1-14, 2014.

27. R. Li, L. Xu, X. Shi, L. Chen and C. Cui, "Two-Dimensional NC-Music DOA Estimation Algorithm with a Conformal Cylindrical Antenna Array", Journal of Electromagnetic Waves and Applications, vol. 25, no. 5-6, pp. 805-818, 2011.

28. Y. Zou, H. Xie, L. Wan, G. Han and W. Li, "2D-DOA and Mutual Coupling Estimation in Vehicle Communication System via Conformal Array", Mobile Information Systems, vol. 2015, pp. 1-10, 2015.

29. Z. Liang, J. Ouyang and F. Yang, "A hybrid GA-PSO optimization algorithm for conformal antenna array pattern synthesis", Journal of Electromagnetic Waves and Applications, vol. 32, no. 13, pp. 1601-1615, 2018.

30. M. Comisso and R. Vescovo, "Fast 3D Pattern Synthesis with Polarization and Dynamic Range Ratio Control for Conformal Antenna Arrays", International Journal of Antennas and Propagation, vol. 2014, pp. 1-9, 2014.

31. R. Karimzadeh Baee, K. Forooraghi and S. Chamaani, "Conformal Array Pattern Synthesis Using a Hybrid WARP/2LB-MOPSO Algorithm", International Journal of Antennas and Propagation, vol. 2012, pp. 1-7, 2012.

32. M. Comisso and R. Vescovo, "Fast Co-Polar and Cross-Polar 3D Pattern Synthesis With Dynamic Range Ratio Reduction for Conformal Antenna Arrays", IEEE Transactions on Antennas and Propagation, vol. 61, no. 2, pp. 614-626, 2013.

33. K. Yang, Z. Zhao, J. Ouyang, Z. Nie and Q. Liu, "Optimisation method on conformal array element positions for low sidelobe pattern synthesis", IET Microwaves, Antennas \& Propagation, vol. 6, no. 6, p. 646, 2012.

34. J. Ouyang, J. Zhang, K. Zhi Zhang, J. Yang, X. Luo and F. Yang, "Analysis and synthesis of conformal conical surface linear phased array with volume surface integral equation+AEP (Active Element Pattern) and INSGA-II", IET Microwaves, Antennas \& Propagation, vol. 6, no. 11, pp. 1277-1285, 2012.

35. Y. Bai, S. Xiao, C. Liu and B. Wang, "A Hybrid IWO/PSO Algorithm for Pattern Synthesis of Conformal Phased Arrays", IEEE Transactions on Antennas and Propagation, vol. 61, no. 4, pp. 2328-2332, 2013.

36. Z. Huang, H. Zhang and J. Zhou, "Full polarimetric sum and difference patterns synthesis for conformal array", Electronics Letters, vol. 51, no. 8, pp. 602-604, 2015.

37. W. Hu, X. Wang, Y. Li and S. Xiao, "Synthesis of Conformal Arrays With Matched Dual-Polarized Patterns", IEEE Antennas and Wireless Propagation Letters, vol. 15, pp. 1341-1344, 2016. 